\title{
Fuzzy Bernstein Type Inequalities
}

\author{
Chun-Gou Zhang \\ School of Mathematical Sciences, \\ Capital Normal University, \\ Beijing 100048, China \\ E-mail: 3773@mail.cnu.edu.cn
}

\author{
Chun-Juan Yang \\ School of Mathematical Sciences, \\ Capital Normal University, \\ Beijing 100048, China \\ E-mail: yangchunjuan125@163.com
}

\begin{abstract}
It is well known that Bernstein type inequalities play as an important role in analysis mathematics and some other areas, and which have been studied extensively and used widely. In this paper, we intend to extend these inequalities to fuzzy mathematics, namely establish fuzzy Bernstein type inequalities.
\end{abstract}

Keywords-fuzzy number; fuzzy function; fuzzy derivative; Bernstein type inequalities

\section{INTRODUCTION}

It is well known that Bernstein type inequalities on derivatives of polynomials play as an important role in analysis mathematics and some other areas, which have been studied extensively and used widely.

Suppose

$$
P_{n}(x)=\sum_{k=0}^{n} a_{k} x^{k}
$$

is a real polynomial of $n$ degree, then a Bernstein type inequality on arbitrary interval $(\alpha, \beta)$ is as follows.

$$
\left|P_{n}^{\prime}(x)\right| \leq \frac{n\left\|P_{n}\right\|_{[\alpha, \beta]}}{\sqrt{(\beta-x)(x-\alpha)}} \quad x \in(\alpha, \beta),
$$

where

$$
\left\|P_{n}\right\|_{\alpha, \beta]}=\sup _{x \in[\alpha, \beta]}|P(x)| \text {. }
$$

In particular, if $\alpha=0$ and $\beta=1$, then the inequality turns into

$$
\| P_{n}^{\prime}(x) \mid \leq \frac{n\left\|P_{n}\right\|_{[0,1]}}{\sqrt{x(1-x)}} \quad x \in(0,1) .
$$

In 1960, Szegö in [1] extended the Bernstein type inequality into the following more generalized form

$$
\begin{aligned}
& (x-\alpha)(\beta-x)\left(P_{n}^{\prime}(x)\right)^{2}+n^{2} P_{n}^{2}(x) \leq n^{2}\left\|P_{n}\right\|_{[\alpha, \beta]}^{2} \\
& x \in[\alpha, \beta] .
\end{aligned}
$$

Obviously, when $\alpha=0, \beta=1$, the inequality becomes into

$$
\begin{aligned}
& x(1-x)\left(P_{n}^{\prime}(x)\right)^{2}+n^{2} P_{n}^{2}(x) \leq n^{2}\left\|P_{n}\right\|_{[0,1]}^{2} . \\
& x \in[0,1] .
\end{aligned}
$$

The main purpose of this paper is to extend these inequalities in fuzzy mathematics, namely establish so-called fuzzy Bernstein type inequalities. In the next section 2 we will introduce some preliminaries of fuzzy mathematics involved in this paper, while our main results will be presented in section 3 .

\section{PRELIMINARIES OF FUZZY MATHEMATICS}

Fuzzy real number (briefly called as fuzzy number) was first introduced by Dubois and Prade (1978) [2], later it was modified by Goetschel and Voxman (1983) [3] as follows

Definition 2.1 A fuzzy number is a fuzzy set

$\mu: \mathbb{R} \rightarrow[0,1]$

with the following properties:

(1) $\mu$ is upper semicontinuous on $\mathbb{R}$, i.e., for any $x_{0} \in \mathbb{R}$ and $\varepsilon>0$, there exists $x_{0}$ neighborhood $\mathrm{V}\left(x_{0}\right)$ such as $\mu(x) \leq \mu\left(x_{0}\right)+\varepsilon$, for each $x \in \mathrm{V}\left(x_{0}\right)$.

(2) $\mu(x)=0$ outside of some interval $[c, d]$

(3) There are real numbers $a$ and $b, c \leq a \leq b \leq d$ such as $\mu$ is increasing on the interval $[c, a]$, decreasing on the interval $[b, d]$ and $\mu(x)=1$ on $[a, b]$.

We denote by $F$ the set of all fuzzy numbers as defined above. A popular definition of fuzzy number from [4] is below

Definition 2.2 Let $\mu: \mathbb{R} \rightarrow[0,1]$ with the following properties:

(1) $\mu$ is normal, i.e., there exists $x_{0} \in \mathbb{R}$ such as

$$
\mu\left(x_{0}\right)=1 \text {. }
$$

(2) for any $x, y \in \mathbb{R}$ and $\lambda \in[0,1]$, there holds

$$
\mu(\lambda x+(1-\lambda) y)) \geq \min \{\mu(x), \mu(y)\} \text {. }
$$

(3) $\mu$ is upper semicontinuous on $\mathbb{R}$, i.e., for any $x_{0} \in \mathbb{R}$ and $\varepsilon>0$, there exists $x_{0}$ neighborhood $\mathrm{V}\left(x_{0}\right)$ such as

$$
\mu(x) \leq \mu\left(x_{0}\right)+\varepsilon \text {, for each } x \in \mathrm{V}\left(x_{0}\right) .
$$

(4) The support set $\operatorname{supp}(\mu)$ is compact in $\mathbb{R}$, where $\operatorname{supp}(\mu)=\{x \in \mathbb{R} \mid \mu(x)>0\}$

We use $F$ to denote the set of all fuzzy numbers as defined here. In fact, the definition 2.1 and 2.2 for fuzzy number is equivalent, That is that we have the following proposition.

Proposition 2.3 F = F . 
Proof Since it is obvious that $F \subset F$, we only need to show that $F \supset F$. According to definition 2.2 (4), we suppose $\overline{\operatorname{supp}(\mu)}=[c, d]$, that is $\mu(x)=0$ for all $x \bar{\in}[c, d]$. Let

$$
\mu^{1}=\{x \in[c, d] \mid \mu(x)=1\},
$$

then $\mu^{1}$ is a close interval (see [5]). Suppose $\mu^{1}=[a, b]$, then obviously $[a, b] \subset[c, d]$. Now let

$$
x, y \in[c, a] \text { and } x \leq y \text {, }
$$

by definition 2.2(2), we have that for any $\lambda \in[0,1]$, there holds that

$$
\mu(\lambda x+(1-\lambda) a) \geq \mu(x) .
$$

Owing to that $y \in[x, a]$, thus there exists a $\lambda \in[0,1]$ such that

$$
\begin{aligned}
& \lambda x+(1-\lambda) a=y, \\
& \text { it follows that } \\
& \mu(y)=\mu(\lambda x+(1-\lambda) a) \geq \mu(x) .
\end{aligned}
$$

The remainder is may proved similarly.

Let $\mu \in \mathrm{F}$, for $r \in[0,1]$ we define that

$$
[\mu]^{r}= \begin{cases}\{x \in \mathbb{R}: \mu(x) \geq r\} & 0<r \leq 1 \\ \operatorname{supp}(\mu) & r=0 .\end{cases}
$$

In [5] and [6], we can find that $[\mu]^{r}$ is a closed and bounded

interval of $\mathbb{R}$, therefore which is determined completely by the endpoints of the interval $[\mu]^{r}$, so we can denote directly a fuzzy number with

$$
\mu=[\mu]^{r}=\left[\mu_{-}^{r}, \mu_{+}^{r}\right] \text {, }
$$

where $0 \leq r \leq 1$ and $\mu_{-}^{r}\left(\mu_{+}^{r}\right)$ is the left(right)endpoint of $[\mu]^{r}$. By means of this representation of fuzzy numbers we can define fuzzy addition and scalar multiplication as below.

Definition 2.4 For $\mu, v \in \mathrm{F}$ with

$$
\mu=[\mu]^{r}=\left[\mu_{-}^{r}, \mu_{+}^{r}\right], v=[v]^{r}=\left[v_{-}^{r}, v_{+}^{r}\right]
$$

$(0 \leq r \leq 1)$ and $c \in \mathbb{R}$.

Let (1) fuzzy addition:

$$
\begin{aligned}
& \mu \oplus v=[\mu \oplus v]^{r}=[\mu]^{r}+[v]^{r} \\
& =\left[\mu_{-}^{r}+v_{-}^{r}, \mu_{+}^{r}+v_{+}^{r}\right] \\
& \text { (2) Fuzzy scalar multiplication: } \\
& c \odot \mu=[c \odot \mu]^{r}=c[\mu]^{r} \\
& =\left\{\begin{array}{l}
{\left[c \mu_{-}^{r}, c \mu_{+}^{r}\right] c \geq 0} \\
{\left[c \mu_{+}^{r}, c \mu_{-}^{r}\right] c \geq 0}
\end{array}\right.
\end{aligned}
$$

Remark 2.5 If for $\mu, v \in \mathrm{F}$, there exists a fuzzy number $\omega$ such that $\mu=v \oplus \omega$, then we call $\omega$ as the fuzzy difference of $\mu$ and $v$, and denote by $\omega=\mu$ ! $v$.

We denote the zero element in $\mathrm{F}$ with $\tilde{0}=\chi_{\{0\}}$ (the characteristic function at $X=0$ ). Since there is no negative element in $\mathrm{F}$, it is not a linear vector space. If being endowed in $\mathrm{F}$ with the following measure $D$, then it becomes a metric space, but not complete (see[3]).

Definition $2.6^{[6]}$ For arbitrary $\mu, v \in \mathrm{F}$ with

$$
\begin{aligned}
& \mu=[\mu]^{r}=\left[\mu_{-}^{r}, \mu_{+}^{r}\right], v=[v]^{r}=\left[v_{-}^{r}, v_{+}^{r}\right] \\
& (0 \leq r \leq 1) . \\
& \text { Let } \\
& D(\mu, v)=\sup _{0 \leq r \leq 1} \max \left\{\left|\mu_{-}^{r}-v_{-}^{r}\right|,\left|\mu_{+}^{r}-v_{+}^{r}\right|\right\} .
\end{aligned}
$$

In particular,

$$
D(\mu, \tilde{0})=\sup _{0 \leq r \leq 1} \max \left\{\left|\mu_{-}^{r}\right|,\left|\mu_{+}^{r}\right|\right\} \text {. }
$$

This measure has the following properties.

Proposition $2.7^{[6]}$ For arbitrary $\mu, v, \omega, \gamma \in \mathrm{F}, \alpha \in R$, there hold

(1) $D(\mu \oplus \omega, v \oplus \omega)=D(\mu, v)$;

(2) $D(\alpha \odot \mu, \alpha \odot v)=|\alpha| D(\mu, v)$;

(3) $D(\mu \oplus v, \omega \oplus \gamma) \leq D(\mu, \omega)+D(v, \gamma)$.

With this measure $D$, we can induce a measure in $\mathrm{F}$ as follows.

Definition $2.8^{[6]}$ For arbitrary $\mu \in \mathrm{F}$, let

$$
\|\mu\|=D(\mu, \tilde{0})=\sup _{0 \leq r \leq 1} \max \left\{\left|\mu_{-}^{r}\right|,\left|\mu_{+}^{r}\right|\right\} \text {. }
$$

We use $\tilde{C}[0,1]$ to denote the space of all real and bounded functions on $[0,1]$ with the following properties

(1) $\quad f(x)$ is left continuous at $x \in(0,1]$;

(2) $f(x)$ exists right limitation at $x \in[0,1)$;

(3) $f(x)$ is right continuous at $x=0$.

Obviously, there holds $C[0,1] \subset \tilde{C}[0,1]$ (where $C[0,1]$ is the space of all continuous functions on $[0,1]$ and endowed with uniform norm). For the set of fuzzy numbers $F$, one can find the following embedding theorem in [7].

Theorem 2.9 For arbitrary $\mu \in \mathrm{F}$, if writing

$$
j(\mu)=\left(\mu_{-}^{r}, \mu_{+}^{r}\right)(0 \leq r \leq 1),
$$

then $j(\mathrm{~F})$ is a close convex cone with the vertex point at the origin and

$$
j: \mathrm{F} \rightarrow \tilde{C}[0,1] \times \tilde{C}[0,1]
$$

satisfying the following conditions:

(1) For arbitrary $\mu, v \in \mathrm{F}, s \geq 0, t \geq 0$, there holds 


$$
j(s \mu+t v)=s j(\mu)+t j(v) .
$$

(2) $D(\mu, v)=\|j(\mu)-j(v)\|$.

From this theorem, we can know that if

$$
\mu_{-}^{r}, \mu_{+}^{r} \in \tilde{C}[0,1] \text { and } \mu_{-}^{r} \leq \mu_{+}^{r} \text { for any } r \in[0,1] \text {, }
$$

then the preimage $j^{-1}\left(\mu_{-}^{r}, \mu_{+}^{r}\right)$ under the embedding map $j$ is a fuzzy number $\mu$ and

$$
\mu(x)=\sup \left\{r \in[0,1]: \mu_{-}^{r} \leq x \leq \mu_{+}^{r}\right\} \quad x \in \mathbb{R},
$$

thus in the sequel we directly denote a fuzzy number $\mu$ with a vector $\left(\mu_{-}^{r}, \mu_{+}^{r}\right)$.

Next, we turn to fuzzy functions.

Definition $2.10^{[6]}$ Suppose $I \subset \mathbb{R}$, then the map

$$
\tilde{f}: I \rightarrow \mathrm{F}
$$

is referred as a fuzzy function on $I$, and let

$$
\tilde{f}(x)=\left(\tilde{f}_{-}^{r}(x), \tilde{f}_{+}^{r}(x)\right)
$$

where $x \in I, r \in[0,1], \tilde{f}_{-}^{r}(x)\left(\tilde{f}_{+}^{r}(x)\right)$ is the left(right) endpoint function of the interval $[\tilde{f}(x)]^{r}$. In particular, for

$$
a_{k}, b_{k} \in \mathrm{F}, k=0,1,2, \cdots, n,
$$

we call

$$
\tilde{P}_{n}(x)=\left(\sum_{k=0}^{n}\right)^{*} a_{k} \odot x^{k}
$$

and

$$
\tilde{T}_{n}(x)=\left(\sum_{k=0}^{n}\right)^{*}\left(a_{k} \odot \cos k x \oplus b_{k} \odot \sin k x\right)
$$

as fuzzy algebraic and trigonometric polynomial of $n$ degree respectively, where $\left(\sum\right)^{*}$ denoting fuzzy sum.

Let $C_{F}(I)$ be the space of all continuous fuzzy functions on $I$, and define the measure

$$
D^{*}(\tilde{f}, \tilde{g})=\sup _{x \in I} D(\tilde{f}(x), \tilde{g}(x)) \text {. }
$$

Specially, let

$$
D^{*}(\tilde{f}, \tilde{0})=\|\tilde{f}\| \text {. }
$$

For a fuzzy function $\tilde{f} \in \mathrm{C}_{\mathrm{F}}(I)$, we define the $\mathrm{H}$ derivative below.

Definition $2.11^{[6]}$ Let $x \in I \subset \mathbb{R}$ and suppose for any sufficiently small positive number $h$ such as

$$
x+h, x-h \in I
$$

the fuzzy difference

$$
\tilde{f}(x+h) ! \tilde{f}(x) \text { and } \tilde{f}(x) ! \tilde{f}(x-h)
$$

exist, if there exists a fuzzy number $\mu \in \mathrm{F}$ such as

$$
\lim _{h \rightarrow 0^{+}} \frac{1}{h} \odot(\tilde{f}(x+h) ! \tilde{f}(x))=\mu
$$

and

$$
\lim _{h \rightarrow 0^{+}} \frac{1}{h} \odot(\tilde{f}(x) ! \tilde{f}(x-h))=\mu,
$$

then we say the fuzzy function $f$ at $x$ H-differentiable and call the fuzzy number $\mu$ as the H-derivative of the fuzzy function $f$ at $x$ denoting by $\mu=f_{H}^{\prime}(x)$, where the limitation is taken under the metrics $D$. Similarly we can define higher order H-derivatives $f_{H}^{(n)}(x)$.

From this definition we can see that H-differentiable is more strict than ordinary differentiable. In this paper, we need the following properties of the $\mathrm{H}$-derivative.

Proposition $2.12^{[6]}$ (1) Suppose

$$
\tilde{f}(x)=x^{n} \odot \mu, \quad x \in \mathbb{R}, \mu \in \mathrm{F} \text { and } n \in N,
$$

then $\tilde{f}(x)$ is H-differentiable on $[0,+\infty)$ and

$$
f_{H}^{\prime}(x)=n x^{n-1} \odot \mu \text {. }
$$

(2) Suppose that fuzzy functions $\tilde{f}, \tilde{g}$ are $\mathrm{H}-$ differentiable, then for arbitrary $\mu, v \in \mathbb{R}$ there holds $(\mu \odot \tilde{f} \oplus \nu \odot \tilde{g})_{H}^{\prime}=\mu \odot \tilde{f}_{H}^{\prime} \oplus \nu \odot \tilde{g}_{H}$

(3) Suppose that $I \subset \mathbb{R}$ is a closed interval, $g: I \rightarrow \mathbb{R}$

is differentiable and strictly increasing, and

$\tilde{f}: g(I) \rightarrow \mathrm{F}$

is H-differentiable, then $(\tilde{f} \circ g)_{H}^{\prime}(x)$ exists and for each $x \in I$, there holds

$$
(\tilde{f} \circ g)_{H}^{\prime}(x)=\tilde{f}_{H}^{\prime}(g(x)) \odot g^{\prime}(x) .
$$

Proposition $2.13^{[5,8]}$ Suppose that $\tilde{f}: I \rightarrow \mathrm{F}$ is a fuzzy function, let

$$
\tilde{f}(x)=\left(\tilde{f}_{-}^{r}(x), \tilde{f}_{+}^{r}(x)\right) \quad x \in I .
$$

If $\tilde{f}(x)$ is H-differentiable, then $\tilde{f}_{-}^{r}(x)$ and $\tilde{f}_{+}^{r}(x)$ are differentiable at $x$ for each $r \in[0,1]$, and

$$
f_{H}^{\prime}(x)=\left(\left(\tilde{f}_{-}^{r}\right)^{\prime}(x),\left(\tilde{f}_{+}^{r}\right)^{\prime}(x)\right) \text {. }
$$

Corollary If $\tilde{f}(x) \in \mathrm{F}$ is H-differentiable of $p$ order at $x$, then $\tilde{f}_{-}^{r}(x)$ and $\tilde{f}_{+}^{r}(x)$ are differentiable of $p$ order at $X$ and

$$
\tilde{f}_{H}^{(p)}(x)=\left(\left(\tilde{f}_{-}^{r}\right)^{(p)}\left((x),\left(\tilde{f}_{+}^{r}\right)^{(p)}(x)\right) .\right.
$$

\section{MAIN RESULTS}

Let

$$
\tilde{P}_{n}(x)=\left(\sum_{k=0}^{n}\right)^{*} a_{k} \odot x^{k} a_{k} \in \mathrm{F}, k=0,1, \cdots, n
$$


be a fuzzy polynomial of $n$ degree, where $\left(\sum\right)^{*}$ is fuzzy sum.

Theorem 3.1 The following fuzzy Bernstein type inequality holds

$$
\left.D\left(\tilde{P}_{n}\right)_{H}^{\prime}(x), \tilde{0}\right) \leq \frac{n\left\|\tilde{P}_{n}\right\|_{[0,1]}}{\sqrt{x(1-x)}} \quad x \in(0,1),
$$

where $\left(\tilde{P}_{n}\right)_{H}^{\prime}(x)$ is the H-derivative of the fuzzy polynomial $\tilde{P}_{n}(x)$.

Proof Let's assume that $a_{k}=\left(\left(a_{k}\right)_{-}^{r},\left(a_{k}\right)_{+}^{r}\right)$, then

$$
\begin{aligned}
& \tilde{P}_{n}(x)=\left(\sum_{k=0}^{n}\right)^{*} a_{k} \odot x^{k}=\sum_{k=0}^{n}\left(\left(a_{k}\right)_{-}^{r},\left(a_{k}\right)_{+}^{r}\right) x^{k} \\
& =\left(\sum_{k=0}^{n}\left(a_{k}\right)_{-}^{r} x^{k}, \sum_{k=0}^{n}\left(a_{k}\right)_{+}^{r} x^{k}\right) \\
& =:\left(\left(\tilde{P}_{n}\right)_{-}^{r}(x),\left(\tilde{P}_{n}\right)_{+}^{r}(x)\right) .
\end{aligned}
$$

By Proposition 2.12 we know that the fuzzy polynomial $\tilde{P}_{n}(x)$ is H-differentiable on $[0,1]$ and

$$
\begin{aligned}
& \left(\tilde{P}_{n}\right)_{H}^{\prime}(x)=\left(\left(\tilde{P}_{n}^{\prime}\right)_{-}^{r}(x),\left(\tilde{P}_{n}\right)_{+}^{r}(x)\right) \\
& =\left(\left(\left(P_{n}\right)_{-}^{r}\right)^{\prime}(x),\left(\left(P_{n}\right)_{+}^{r}\right)^{\prime}(x)\right) .
\end{aligned}
$$

Using the Bernstein type inequality, for any $r \in[0,1]$ we have

$$
\begin{aligned}
& \left|\left(\tilde{P}_{n}^{\prime}\right)_{-}^{r}(x)\right|=\left|\left(\left(P_{n}\right)_{-}^{r}\right)^{\prime}(x)\right| \leq \frac{n\left\|\left(\tilde{P}_{n}\right)_{-}^{r}\right\|_{[0,1]}}{\sqrt{x(1-x)}} \\
& x \in(0,1)
\end{aligned}
$$

and

$$
\begin{aligned}
& \left|\left(\tilde{P}_{n}^{\prime}\right)_{+}^{r}(x)\right|=\left|\left(\left(P_{n}\right)_{+}^{r}\right)^{\prime}(x)\right| \leq \frac{n\left\|\left(\tilde{P}_{n}\right)_{+}^{r}\right\|_{[0,1]}}{\sqrt{x(1-x)}} \\
& x \in(0,1) .
\end{aligned}
$$

It is follows that for $x \in[0,1], r \in[0,1]$ there holds

$$
\begin{aligned}
& D\left(\left(\tilde{P}_{n}\right)_{H}^{\prime}(x), \tilde{0}\right) \\
= & \sup _{0 \leq r \leq 1} \max \left\{\left|\left(\tilde{P}_{n}^{\prime}\right)_{-}^{r}(x)\right|,\left|\left(\tilde{P}_{n}^{\prime}\right)_{+}^{r}(x)\right|\right\} \\
\leq & \sup _{0 \leq r \leq 1} \max \left\{\frac{n\left\|\left(\tilde{P}_{n}\right)_{-}^{r}\right\|_{[0,1]}}{\sqrt{x(1-x)}}, \frac{n\left\|\left(\tilde{P}_{n}\right)_{+}^{r}\right\|_{[0,1]}}{\sqrt{x(1-x)}}\right\} \\
= & \frac{n\left\|\tilde{P}_{n}\right\|_{0,1]}}{\sqrt{x(1-x)}} .
\end{aligned}
$$

The proof is completed.

Corollary For any closed interval $[\alpha, \beta] \subset[0, \infty)$, the following inequality holds that

$$
D\left(\left(\tilde{P}_{n}\right)_{H}^{\prime}(x), \tilde{0}\right) \leq \frac{\left\|n \tilde{P}_{n}\right\|_{[\alpha, \beta]}}{\sqrt{(x-\alpha)(\beta-x)}}
$$

$x \in(\alpha, \beta)$.

Proof Let

$$
\sqrt{ }
$$

$x=(\beta-\alpha) t+\alpha, t \in[0,1]$,

obviously which is strictly increasing, thus we have

$\tilde{P}_{n}(x)=\tilde{P}_{n}((\beta-\alpha) t+\alpha), \quad x \in[\alpha, \beta]$.

Writing

$$
\tilde{t}_{n}(t)=\tilde{P}_{n}((\beta-\alpha) t+\alpha), \quad t \in[0,1],
$$

then by Proposition 2.12 (3) we can get

$$
\left(\tilde{P}_{n}\right)_{H}^{\prime}(x)=\frac{1}{\beta-\alpha} \odot\left(\tilde{t}_{n}\right)_{H}^{\prime}(t), t \in[0,1] .
$$

On account of

$\left\|\tilde{t}_{n}\right\|_{[0,1]}=\left\|\tilde{P}_{n}\right\|_{\alpha, \beta]}$

and

$$
D\left(\left(\tilde{t}_{n}\right)_{H}^{\prime}(t), \tilde{0}\right) \leq \frac{n\left\|\tilde{t}_{n}\right\|_{[0,1]}}{\sqrt{t(1-t)}} \quad t \in(0,1),
$$

therefor for arbitrary $x \in(\alpha, \beta)$, we have

$$
\begin{aligned}
& D\left(\left(\tilde{P}_{n}\right)_{H}^{\prime}(x), \tilde{0}\right)=\frac{1}{\beta-\alpha} D\left(\left(\tilde{t}_{n}\right)_{H}^{\prime}(t), \tilde{0}\right) \\
\leq & \frac{1}{\beta-\alpha} \frac{\left.n \| \tilde{t}_{n}\right) \|_{[0,1]}}{\sqrt{t(1-t)}} \$ \\
= & \frac{n\left\|\tilde{P}_{n}\right\|_{\alpha, \beta]}}{\sqrt{(x-\alpha)(\beta-x)}} .
\end{aligned}
$$

That is the proof of this corollary.

Similar to the extension of Bernstein type inequalities as stated in section 1 above, now we extend the fuzzy Bernstein type inequalities.

Theorem 3.2 Suppose $\tilde{P}_{n}(x)$ is a fuzzy polynomial of $n$ degree, then for arbitrary $x \in[0,1]$ the following inequality holds

$$
\begin{aligned}
& x(1-x)\left(D\left(\left(\tilde{P}_{n}\right)_{H}^{\prime}(x), \tilde{0}\right)\right)^{2}+n^{2}\left(D\left(\tilde{P}_{n}(x), \tilde{0}\right)^{2}\right. \\
& \leq 2 n^{2}\left\|\tilde{P}_{n}\right\|_{0,1]}^{2}
\end{aligned}
$$

Proof According to the generalized form of the Bernstein type inequality which have been stated in section 1 above, for arbitrary $x \in[0,1], r \in[0,1]$ we have

$$
\begin{aligned}
& x(1-x)\left|\left(\left(\tilde{P}_{n}\right)_{-}^{r}\right)^{\prime}(x)\right|^{2}+n^{2}\left(\left(\tilde{P}_{n}\right)_{-}^{r}\right)^{2}(x) \\
& \leq n^{2}\left\|\tilde{P}_{n}\right\|_{0,1]}^{2} \\
& \text { and } \\
& x(1-x)\left|\left(\left(P_{n}\right)_{+}^{r}\right)^{\prime}(x)\right|^{2}+n^{2}\left(\left(\tilde{P}_{n}\right)_{+}^{r}\right)^{2}(x) \\
& \leq n^{2}\left\|\tilde{P}_{n}\right\|_{[0,1]}^{2},
\end{aligned}
$$


it follows that for arbitrary $x \in[0,1], r \in[0,1]$ there hold

$$
\begin{aligned}
& x(1-x)\left|\left(\left(\tilde{P}_{n}\right)_{-}^{r}\right)^{\prime}(x)\right|^{2} \leq n^{2}\left\|\tilde{P}_{n}\right\|_{[0,1]}^{2}, \\
& n^{2}\left(\left(\tilde{P}_{n}\right)_{-}^{r}\right)^{2}(x) \leq n^{2}\left\|\tilde{P}_{n}\right\|_{[0,1]}^{2} \\
& \text { and } \\
& x(1-x)\left|\left(\left(\tilde{P}_{n}\right)_{+}^{r}\right)^{\prime}(x)\right|^{2} \leq n^{2}\left\|\tilde{P}_{n}\right\|_{[0,1]}^{2}, \\
& n^{2}\left(\left(\tilde{P}_{n}\right)_{+}^{r}\right)^{2}(x) \leq n^{2}\left\|\tilde{P}_{n}\right\|_{[0,1]}^{2} .
\end{aligned}
$$

Recalling

$$
\begin{aligned}
& \left(\tilde{P}_{n}\right)_{H}^{\prime}(x)=\left(\left(\tilde{P}_{n}^{\prime}\right)_{-}^{r}(x),\left(\tilde{P}_{n}^{\prime}\right)_{+}^{r}(x)\right) \\
& =\left(\left(\left(P_{n}\right)_{-}^{r}\right)^{\prime}(x),\left(\left(P_{n}\right)_{+}^{r}\right)^{\prime}(x)\right),
\end{aligned}
$$

therefore we have that for arbitrary $x \in[0,1]$,

$$
\begin{aligned}
& x(1-x) \sup _{0 \leq r \leq 1} \max \left\{\mid\left(\left.\left(\tilde{P}_{n}^{\prime}\right)_{-}^{r}(x)\right|^{2}, \mid\left(\left.\left(\tilde{P}_{n}^{\prime}\right)_{+}^{r}(x)\right|^{2}\right\}\right.\right. \\
& \leq n^{2}\left\|\tilde{P}_{n}\right\|_{[0,1]}^{2}
\end{aligned}
$$

and

$$
\begin{aligned}
& \sup _{0 \leq r \leq 1} \max \left\{\mid\left(\left.\left(\tilde{P}_{n}\right)_{-}^{r}(x)\right|^{2}, \mid\left(\left.\left(\tilde{P}_{n}\right)_{+}^{r}(x)\right|^{2}\right\}\right.\right. \\
& \leq n^{2}\left\|\tilde{P}_{n}\right\|_{[0,1]}^{2} .
\end{aligned}
$$

Adding the two sides of the two inequalities above respectively, which will complete the proof of Theorem 3.2.

Using the same technique as in the proof of Corollary of Theorem 3.1, it is no difficult to show the following conclusion.
Corollary Suppose $[\alpha, \beta] \subset[0, \infty)$, then for arbitrary $x \in[\alpha, \beta]$ we have

$$
\begin{aligned}
& (x-\alpha)(\beta-x)\left(D\left(\left(\tilde{P}_{n}\right)_{H}^{\prime}(x), \tilde{0}\right)\right)^{2}+n^{2}\left(D\left(\tilde{P}_{n}(x), \tilde{0}\right)^{2}\right. \\
& \leq 2 n^{2}\left\|\tilde{P}_{n}\right\|_{[0,1]}^{2}
\end{aligned}
$$

Remark 3.3 It still is an open problem whether or not the constant 2 of the right side in above inequality can be improved into 1.

\section{ACKNOWLEDGMENT}

This project is supported by the Natural Science Foundation of China (10971141, 11271263) and by Beijing Municipal Education Commission science and technology plan project (KM201510028003).

\section{REFERENCES}

[1] G. Szegö, über zinen satz des Herrn serge Bernstein, Schriften Königsberger Gelehrten Ges. Naturwiss. Kl.5 (1928-29), 59-70.

[2] D.Dubois and H.Prade, Operations on fuzzy numbers, Internat. J. of Systems Sci. 9 (1978), 613-626.

[3] R. Goetschel and W. Voxman, Topological properties of fuzzy numbers, Fuzzy Sets and Systems 9(1983), 87-99.

[4] V. Totik, Approximation by Meyer-Köing and Zeller type operators, Math. Z. 184(1983), 425-446.

[5] R. Goetschel, Jr. and W. Voxman, Elementary fuzzy calculus, Fuzzy Sets and Systems 18(1986), 31-43.

[6] George A. Anastassion, Fuzzy Mathematics: Approximation Theory, Springer-Verlag Berlin Heidelberg, 2010.

[7] Congxin Wu and Ming Ma, On embedding problem of fuzzy number space: Part 1, Fuzzy Sets and Systems 44(1991), 33-38.

[8] O.Kaleva, Fuzzy differential equation, Fuzzy Sets and Systems 24(1987), 301-317. 